\title{
ON FREE SUBSEMIGROUPS OF SKEW FIELDS
}

\author{
L. MAKAR-LIMANOV ${ }^{1}$
}

\begin{abstract}
I will prove in this note that the multiplicative group of a skew field with uncountable center either contains a free nonabelian subsemigroup or is commutative.
\end{abstract}

Let $T$ be a skew field with center $Z$ and let us suppose that $Z$ is an uncountable set. Then the following statement is true.

THEOREM. If $T \neq Z$ then the multiplicative group of $T$ contains a free subsemigroup with two generators.

The proof of the theorem is broken into the following three lemmas.

LEMMA 1. Suppose $T$ has no such subsemigroup. Then for any pair of elements $x, y$ from $T$ there exists a semigroup relation $A(a, b)=B(a, b)$ such that this relation is valid for every pair $x+c, y+d x$, where $c, d \in Z$, and that $\operatorname{deg}_{a} A=$ $\operatorname{deg}_{a} B, \operatorname{deg}_{a} A=\operatorname{deg}_{b} B$.

PROOF. Let us fix a pair $x, y$ and consider all pairs $x+c, y+d x$. Each pair fails to span a free semigroup (with two generators) so it satisfies some relation which of course depends on $c$ and $d$. Now we have only a countable number of possible relations. This means that if we fix, for example, $c=c_{0}$ then there exists a relation which is satisfied by an infinite number of pairs $x+c_{0}, y+d x$. Let us represent this relation as a polynomial in $d$ with coefficients depending on $x, y$ and $c_{0}$. This polynomial has infinitely many roots so all of its coefficients must be zeros (we can use Vandermond's determinant with entries $\left\{d_{i}^{j}\right\}$ ). So this relation is satisfied by the pair $x+c_{0}, y+d x$ for every $d$ in $Z$. Thus for every $c$ there exists a relation $P(c)$ of this type which is satisfied by every pair $x+c, y+d x$ (where $c$ is fixed and $d$ can be taken arbitrarily). Let us call such relations good for $x, y$ and $c$. Among the countably many good relations we can find a relation $P$ which is satisfied by $x+c, y+d x$ for infinitely many $c$ (and any $d$ ). Then the same reasoning shows that this relation $P$ is satisfied by $x+c, y+d x$ for every $c, d$ (because for every fixed $d$ this relation is satisfied for infinitely many $c$ and thus for every $c$ ). Let us call such relations better for $x, y$. Now for each pair $e x, f y$, where $e, f \in Z$, there exists a better relation for $e x, f y$ and again we can see that there exists a better relation which is satisfied by every pair $e x, f y$. By factoring out $e$ and $f$ we can see that this relation has the same degrees in $a$ and in $b$ on its left and right sides.

Received by the editors May 13, 1983 and, in revised form, September 22, 1983.

1980 Mathematics Subject Classification. Primary 16A39, 16A70, 16A25; Secondary 20M05, $20 \mathrm{M} 99$.

${ }^{1}$ The author is supported by NSF Grant No. MCS-8201115. 
LEMMA 2. If the relation $A(a, b)=B(a, b)$ is nontrivial and satisfies the conclusion of Lemma 1 for a pair $x, y \in T$ then there exists some $n=n(x, y)$ such that $\left(a d_{x}\right)^{n} y=0$, where $a d_{x} y=[x, y]=x y-y x$.

Proof. We can write $A(a, b+d a)$ as $\sum_{j} A_{j}(a, b) d^{J-j}$, where $\operatorname{deg}_{b} A_{j}=j$. Let us show first that $A_{1}(a, b)$ uniquely defines $A(a, b)$ if $\operatorname{deg}_{b} A \neq 0$. Suppose that $A(a, b)=a^{i_{1}} b^{j_{1}} \cdots a^{i_{k}} b^{j_{k}}$. Then $A_{1}(a, b)=\sum_{m \in M} a^{m} b a^{I+J-m-1}$, where $I=$ $\sum i_{s}, J=\sum j_{s}$. The set $M$ can be represented as the union of the longest possible arithmetic progressions with difference equal to one. The first of these progressions starts with $i_{1}$ and contains $j_{1}$ terms, the second one starts with $i_{1}+j_{1}+i_{2}$ and contains $j_{2}$ terms, the $m$ th progression starts with $i_{1}+j_{1}+\cdots+j_{m-1}+i_{m}$ and contains $j_{m}$ terms. This information, together with the known $\sum\left(i_{s}+j_{s}\right)$, uniquely defines all $i$ 's and $j$ 's.

Now we may assume that both $\operatorname{deg}_{a} A$ and $\operatorname{deg}_{b} A$ are nonzero because otherwise the relation is trivial. So $A_{1}=B_{1}$ is a nontrivial relation. We can rewrite this relation as the equivalent relation

$$
\sum_{s=0}^{I+J} k_{s} a^{s} b a^{I+J-s}=0 .
$$

(Here all the $k_{s}$ are 0 or \pm 1 but we are going to use only that $k_{s} \in Z$.) We can rewrite relation $(*)$ as

$$
\sum_{s=0}^{I+J-1} k_{s}^{\prime} a^{s} b_{1} a^{I+J-1-s}+k_{I+J}^{\prime} a^{I+J} b=0,
$$

where $b_{1}=[b, a]$. Now $k_{I+J}^{\prime} c^{I+J} y=0$ which means that $k_{I+J}^{\prime}=0$. So instead of the relation $(*)$ we obtain the relation

$$
\sum_{s} k_{s}^{\prime} a^{s} b a^{I+J-1-s}=0
$$

of degree smaller than the degree of relation $(*)$ and which is satisfied by every pair $a=x+c, b=[y, x]$. Now we may apply induction on the degree $\operatorname{deg}_{a}$ of the relation. The inductive hypothesis is that from every relation of type $(*)$ which is satisfied by all pairs $a=u+c, b=v$, where $u$ and $v$ are any fixed elements of $T$ and $c$ is a nonfixed element of $Z$, it follows that $\left(a d_{u}\right)^{n} v=0$ for some $n$. The base of induction, when this degree is one, is trivial. For the induction step we remark that if we start with a nontrivial relation then we obtain a nontrivial relation again because in $(*)$ and $(* *)$ the first nonzero coefficients $k_{s}$ and $k_{s}^{\prime}$ have the same subscript and are equal to each other. This finishes the proof of this lemma.

Now if we assume that $T$ does not contain a free subsemigroup with two generators, we can deduce with the help of Lemmas 1 and 2 that for any pair $x, y \in T$ there exists $n=n(x, y)$ such that $\left(a d_{x}\right)^{n} y=0$. If $T$ is not a field, let us take a pair $x, y$ which does not commute. Let us denote $\left(a d_{x}\right)^{n-2} y\left(\left(a d_{x}\right)^{n-1} y\right)^{-1}$ by $w$ (here $n>1$ is the minimal power of $a d_{x}$ which annihilates $\left.y\right)$. It is clear that $[x, w]=1$. Let us denote the skew subfield spanned by $x$ and $w$ over $Z$ by $D$. The proof of the theorem will be finished if we prove the following lemma. 
LEMMA 3. The skew field $D$ contains a free semigroup with two generators.

PROOF. If $D$ does not contain such a semigroup, then by Lemma 2 for the elements $t=x w$ and $w$ there exists an $n$ such that $\left(a d_{t}\right)^{n} w=0$. But $[t, w]=w$ so $\left(a d_{t}\right)^{n} w=w$ for any $n$. This contradiction shows that $D$ contains a desired subgroup and so does $T$.

REMARK 1. It is possible to prove that every skew field which is finite dimensional over its center and does not coincide with its center contains a noncommutative free subgroup. (This result can be extracted from the papers of A. Lichtman [1 and 2], see also [3].) So the following conjecture sounds quite reasonable: every skew field which is really skew contains a noncommutative free subsemigroup.

REMARK 2. The theorem stated can be looked at as a generalization of a result of I. Kaplansky [4] although the restriction on the cardinality of the center somewhat spoils the picture.

\section{REFERENCES}

1. A. Lichtman, Normal subgroups of the multiplicative group of a skew field, Soviet Math. Dokl. 153 (1963), 1425-1429.

2. Soc. 71 (1978), 174-178.

3. L. Makar-Limanov, On free subgroups of skew fields, preprint.

4. I. Kaplansky, A theorem on division rings, Canad. J. Math. 3 (1951), 290-292.

Department of Mathematics, Wayne State University, Detroit, Michigan 48202 